\title{
The Mean Stability Criteria in terms of Two Measures for Stochastic Differential Equations with Coefficient's Uncertainty
}

\author{
Rui Zhang, ${ }^{1}$ Yinjing Guo, ${ }^{1}$ Xiangrong Wang, ${ }^{2}$ and Xueqing Zhang ${ }^{3}$ \\ ${ }^{1}$ College of Electronic Communication and Physics, Shandong University of Science and Technology, Qingdao 266590, China \\ ${ }^{2}$ College of Mathematics and Systems Science, Shandong University of Science and Technology, Qingdao 266590, China \\ ${ }^{3}$ College of Environmental Science and Engineering, Ocean University of China, Qingdao 266100, China
}

Correspondence should be addressed to Xueqing Zhang; zxq@ouc.edu.cn

Received 21 April 2015; Revised 23 July 2015; Accepted 9 August 2015

Academic Editor: Son Nguyen

Copyright (C) 2015 Rui Zhang et al. This is an open access article distributed under the Creative Commons Attribution License, which permits unrestricted use, distribution, and reproduction in any medium, provided the original work is properly cited.

\begin{abstract}
This paper extends the stochastic stability criteria of two measures to the mean stability and proves the stability criteria for a kind of stochastic Itô's systems. Moreover, by applying optimal control approaches, the mean stability criteria in terms of two measures are also obtained for the stochastic systems with coefficient's uncertainty.
\end{abstract}

\section{Introduction}

Lyapunov's method, which makes an essential use of auxiliary functions (also called Lyapunov functions), is an important approach to study the stability of differential systems including ordinary differential equations (ODEs) and stochastic differential equations (SDEs). This method started in Lyapunov's original work in 1892 [1] for demonstrating stability of ODEs. In the 1960s, Movchan [2] studied the stability with two measures; such works were also developed and can be seen in [3]. In the past decades, Lyapunov's method is modified to the study of stability of Markovian processes [4], stochastic differential systems based on Brownian motions [5], semimartingales [6], or Lévy processes [7] and is also developed with the form of exponential stability [8] or LaSalle theorem [9], and so forth. Recently, the stability for systems with unknown parameters is also discussed [10], and the theorems of stability are widely applied in aerospace [11], state-feedback control [12], automatic control [13], neural networks [14], and other fields.

In this paper, we will discuss the following stochastic Itô's systems:

$$
d x=f(t, x) d t+\sigma(t, x) d B_{t}
$$$$
x\left(t_{0}\right)=x_{0}, t \geq t_{0} \in \mathscr{R}_{+},
$$

where $f, \sigma$ satisfy the usual Lipschitzian conditions and $B$ is $d$-dimensional standard Brownian motion. It is well known that, for a stochastic process $x(t)$ and a given positive function $h(t, x), \lim _{t \rightarrow \infty} h(t, x(\omega, t))=0$ almost surely discussed in [15] does not imply that $\lim _{t \rightarrow \infty} \mathbb{E} h(t, x(t)) \rightarrow 0$. So, we extend Lyapunov's methods used by [3] for ODEs to the stochastic cases and study the mean stability criteria in terms of two measures for system (1).

This paper is organized as follows: In Section 2, we first introduce Lyapunov's derivatives for (1) and deduce the basic comparison results in terms of Lyapunov's function. In Section 3, we prove the stochastic two-measure stability criteria for Itô systems, which can be seen as the extension of that of the ODEs. As described in [16], stability, robustness, and optimality can be considered systematically and simultaneously. In Section 4, the optimal control approach is extended to the stochastic systems with coefficient's uncertainty.

\section{Basic Comparison Results for Stochastic Differential Equations}

Let $(\Omega, \mathscr{F}, P)$ be a given completed probability space, and $\left\{B_{t}\right\}_{t \geq 0}$ is a standard Brownian motion with filtration:

$$
\mathscr{F}_{t}=\sigma\left(B_{s}, s \leq t\right) .
$$


Let $f \in C\left(\mathscr{R}_{+} \times \mathscr{R}^{n}, \mathscr{R}^{n}\right), \sigma \in C\left(\mathscr{R}_{+} \times \mathscr{R}^{n}, \mathscr{R}^{n} \times \mathscr{R}^{d}\right)$ be deterministic functions and satisfy the following Lipschitz condition and linear growth condtion: there exists $L>0$, for every $x, y \in \mathscr{R}^{n}$, such that

$$
\begin{aligned}
& \|f(t, x)-f(t, y)\|+\|\sigma(t, x)-\sigma(t, y)\| \leq L\|x-y\|, \\
& \|f(t, x)\| \leq L(1+\|x\|) .
\end{aligned}
$$

For a given function $V \in C\left(\mathscr{R}_{+} \times \mathscr{R}^{n}, \mathscr{R}\right),(t, x) \in \mathscr{R}_{+} \times \mathscr{R}^{n}$, we denote

$$
\begin{aligned}
& \mathscr{D}^{+} V(t, x)_{(1)}=\limsup _{h \rightarrow 0^{+}} \frac{1}{h} \\
& \cdot \mathbb{E}[V(t+h, x+h f(t, x)+\sqrt{h} \sigma(t, x) \xi) \\
& \quad-V(t, x)],
\end{aligned}
$$

where $\xi$ represents a $d$-dimensional random variable with standard normal distribution; that is, $\xi \sim N(0, I)$ and $I$ is a $d$-order identity matrix.

Remark 1. We use the notation $\mathscr{D}^{+} V(t, x)_{(1)}$ to emphasize the definition with respect to system (1). For convenience, we use the shortened form $\mathscr{D}^{+} V$ to substitute $\mathscr{D}^{+} V(t, x)_{(1)}$.

Remark 2. If $V \in C^{1,2}\left(\mathscr{R}_{+} \times \mathscr{R}^{n}, \mathscr{R}_{+}\right), \mathscr{D}^{+} V(t, x)$ is Lyapunov's operator $\mathscr{L}$ associated with (1); that is,

$$
\mathscr{D}^{+} V_{(1)}=\mathscr{L} V:=V_{t}+\left\langle V_{x}, f\right\rangle+\frac{1}{2}\left\langle\sigma \sigma^{T}, V_{x x}\right\rangle
$$

where $V_{t}$ is the partial derivative for $t, V_{x}$ is the gradient of $V$ for $x$, and $V_{x x}$ is the Hessian matrix of $V$ for $x$.

Since (4) is dependent on expectation calculating, it is not easy to check whether $\mathscr{D}^{+} V$ exists or not. The following lemma gives a condition for the existence of $\mathscr{D}^{+} V$.

Lemma 3. Let $V \in C\left(\mathscr{R}_{+} \times \mathscr{R}^{n}, \mathscr{R}\right)$ be continuous one-order differentiable for $t$ and also satisfy the following condition:

$$
\begin{array}{r}
|V(t, x+y)-V(t, x)-\langle\alpha(t, x), y\rangle| \leq K(t)\|y\|^{2}, \\
\forall x, y \in \mathscr{R}^{n},
\end{array}
$$

where $\alpha(t, x)$ is continuous on $\mathscr{R}_{+} \times \mathscr{R}^{n}$ and $K(t) \geq 0$ is locally bounded for $t$. Then $\mathscr{D}^{+} V(t, x)$ exists at $(t, x)$.

Proof. Denote $y=h f(t, x)+\sqrt{h} \sigma(t, x) \xi$; then, for fixed $t, x$,

$$
\begin{aligned}
\frac{1}{h} \mathbb{E} & {[V(t+h, x+y)-V(t, x)] } \\
= & \frac{1}{h} \mathbb{E}[V(t+h, x+y)-V(t+h, x)] \\
& +\frac{1}{h}[V(t+h, x)-V(t, x)] .
\end{aligned}
$$

By (6) and distribution of $\xi$, we have the first item of the right side of (7) that is bounded:

$$
\begin{aligned}
& \langle\alpha(t, x), f(t, x)\rangle \\
& -K(t, x, y) \mathbb{E}\|\sqrt{h} f(t, x)+\sigma(t, x) \xi\|^{2} \\
& \quad \leq \frac{1}{h} \mathbb{E}[V(t+h, x+y)-V(t+h, x)] \\
& \quad \leq\langle\alpha(t, x), f(t, x)\rangle \\
& -K(t) \mathbb{E}\|\sqrt{h} f(t, x)+\sigma(t, x) \xi\|^{2} .
\end{aligned}
$$

Since $V$ is differentiable for $t$, so the last item of the right side of (7) is also bounded. Therefore, the supremum limit of (7) exists; that is, $\mathscr{D}^{+} V(t, x)$ exist exactly.

The following lemmas will be used later.

Lemma 4 (see Theorem 7.1.2 in [6], or Theorems 4.3 and 4.4 in [17]). Suppose $f, \sigma$ satisfy (3). Then, stochastic differential equation (1) admits a unique strong solution $\{x(t), t \geq 0\}$ such that, for any $h>0([t, t+h] \subset[0, T], l \geq 2)$, there exists $\left(K_{T}\right.$ is a constant dependent only on $T, l$, and $L$ ):

$$
\begin{aligned}
& \mathbb{E} \sup _{t \leq s \leq t+h}\|x(s)\|^{l} \leq K_{T}\left(1+E\|x(t)\|^{l}\right), \\
& \mathbb{E}\|x(r)-x(s)\|^{l} \leq K_{T}\left(1+E\|x(s)\|^{l}\right)|r-s|^{l / 2} .
\end{aligned}
$$

Lemma 5. Suppose $V$ satisfies (6), and $x(t)$ is the solution of (1); let $m(t)=\mathbb{E} V(t, x(t))$; then

$$
D^{+} m(t) \leq \mathbb{E} \mathscr{D}^{+} V(t, x(t)),
$$

where $\mathscr{D}^{+} V(t, x(t))=\left.\mathscr{D}^{+} V(t, x)\right|_{x=x(t)}$ and $D^{+} m(t)$ is the usual right upper Dini derivative defined by

$$
D^{+} m(t)=\limsup _{h \rightarrow 0^{+}} \frac{m(t+h)-m(t)}{h} .
$$

Proof. For small $h>0$, we have

$$
\begin{aligned}
& m(t+h)-m(t)=\mathbb{E}[V(t+h, x(t+h))-V(t \\
& \left.\quad+h, x(t)+h f(t, x(t))+\sigma(t, x(t))\left(B_{t+h}-B_{t}\right)\right) \\
& \quad+V(t+h, x(t)+h f(t, x(t)) \\
& \left.\left.\quad+\sigma(t, x(t))\left(B_{t+h}-B_{t}\right)\right)-V(t, x(t))\right] .
\end{aligned}
$$

We now prove that the first two items of the right side are the higher infinitesimal of $h$. By (1), we know that

$$
\begin{aligned}
\widehat{x}:= & x(t+h) \\
& -\left(x(t)+h f(t, x(t))+\sigma(t, x(t))\left(B_{t+h}-B_{t}\right)\right) \\
= & \int_{t}^{t+h}[f(s, x(s))-f(t, x(t))] d s \\
& +\int_{t}^{t+h}[\sigma(s, x(s))-\sigma(t, x(t))] d B s .
\end{aligned}
$$


For convenience, we denote $\widehat{f}_{s}:=f(s, x(s))-f(t, x(t))$, similar meaning for $\widehat{\sigma}_{s}$. We have

$$
\begin{aligned}
& \mathbb{E}\|\widehat{x}\|^{2} \leq 2\left(\mathbb{E}\left\|\int_{t}^{t+h} \widehat{f}_{s} d s\right\|^{2}+\mathbb{E}\left\|\int_{t}^{t+h} \widehat{\sigma}_{s} d B s\right\|^{2}\right) \\
& \leq 2\left[h \int_{t}^{t+h} \mathbb{E}\left\|\hat{f}_{s}\right\|^{2} d s+\int_{t}^{t+h} \mathbb{E}\left\|\widehat{\sigma}_{s}\right\|^{2} d s\right] \\
& \leq 2 L\left[h \int_{t}^{t+h} \mathbb{E}\|x(s)-x(t)\|^{2} d s\right. \\
& \left.+\int_{t}^{t+h} \mathbb{E}\|x(s)-x(t)\|^{2} d s\right] .
\end{aligned}
$$

By Lemma 4 and inequality (10), we have

$$
\mathbb{E}\|\widehat{x}\|^{2} \leq 2 L K_{T}\left(1+\mathbb{E}\|x(t)\|^{2}\right)\left(\frac{1}{2} h^{3}+h^{2}\right) .
$$

Let $\widetilde{x}_{t}:=x(t)+h f(t, x(t))+\sigma(t, x(t))\left(B_{t+h}-B_{t}\right)$; then $(\alpha(x)$ replaces $\alpha(t, x)$ for shortening)

$$
\begin{aligned}
& \mathbb{E}\left|V(t+h, x(t+h))-V\left(t+h, \tilde{x}_{t}\right)-\left\langle\alpha\left(\tilde{x}_{t}\right), \hat{x}\right\rangle\right| \\
& \quad \leq C(h) h^{2} .
\end{aligned}
$$

Now we estimate the order of

$$
\begin{aligned}
\mathbb{E}\left\langle\alpha\left(\tilde{x}_{t}\right), \hat{x}\right\rangle= & \mathbb{E}\langle\alpha(x(t)), \hat{x}\rangle \\
& +\mathbb{E}\left\langle\alpha\left(\tilde{x}_{t}\right)-\alpha(x(t)), \hat{x}\right\rangle .
\end{aligned}
$$

Since $\mathbb{E}\langle\alpha(x(t)), \hat{x}\rangle=\mathbb{E}\left\langle\alpha(x(t)), \mathbb{E}\left[\widehat{x} \mid \mathscr{F}_{t}\right]\right\rangle$ and $\mathbb{E}\left[\widehat{x} \mid \mathscr{F}_{t}\right]=$ $\mathbb{E}\left[\int_{t}^{t+h} \widehat{f}_{s} d s \mid \mathscr{F}_{t}\right]$, so

$$
\begin{aligned}
\mid \mathbb{E} & \langle\alpha(x(t)), \hat{x}\rangle|=| \mathbb{E}\left\langle\alpha(x(t)), \int_{t}^{t+h} \widehat{f}_{s} d s\right\rangle \mid \\
& \leq C_{1}(h) h^{3 / 2}, \\
\mid \mathbb{E} & \left\langle\alpha\left(\widetilde{x}_{t}\right)-\alpha(x(t)), \widehat{x}\right\rangle \mid \leq\left\|\alpha\left(\widetilde{x}_{t}\right)-\alpha(x(t))\right\|\|\widehat{x}\| \\
& \leq C_{2}(h) h^{3 / 2},
\end{aligned}
$$

where $C_{1}(h), C_{2}(h)$ are continuous positive functions. By (19), we have

$$
\left|\mathbb{E}\left\langle\alpha\left(\tilde{x}_{t}\right), \widehat{x}\right\rangle\right| \leq C_{3}(h) h^{3 / 2} .
$$

Since

$$
\begin{aligned}
\mid \mathbb{E} & {\left[V(t+h, x(t+h))-V\left(t+h, \tilde{x}_{t}\right)\right] \mid } \\
\leq & \mathbb{E}\left|V(t+h, x(t+h))-V\left(t+h, \widetilde{x}_{t}\right)-\left\langle\alpha\left(\widetilde{x}_{t}\right), \widehat{x}\right\rangle\right| \\
& +\left|\mathbb{E}\left\langle\alpha\left(\widetilde{x}_{t}\right), \hat{x}\right\rangle\right|,
\end{aligned}
$$

by (17) and (20), we see that the first two items of right side are higher infinitesimal of $t$. So we have

$$
\lim _{h \rightarrow 0^{+}} \frac{1}{h}\left|\mathbb{E}\left[V(t+h, x(t+h))-V\left(t+h, \tilde{x}_{t}\right)\right]\right|=0 .
$$

For the last two items of (13), since $x(t)$ is independent of $B_{t+h}-B_{t}$ with normal distribution $N(0, h)$, so we have

$$
\begin{aligned}
& \limsup _{h \rightarrow 0} \frac{1}{h} \mathbb{E}[V(t+h, x(t)+h f(t, x(t)) \\
& \left.\left.\quad+\sigma(t, x(t))\left(B_{t+h}-B_{t}\right)\right)-V(t, x(t))\right] \\
& \quad \leq \mathbb{E}\left\{\limsup _{h \rightarrow 0} \frac{1}{h}\right. \\
& . \mathbb{E}\left[V\left(t+h, y+h f(t, y)+\sigma(t, y)\left(B_{t+h}-B_{t}\right)\right)\right. \\
& \left.-V(t, y)]_{y=x(t)}\right\}=\mathbb{E}\left[\left.\mathscr{D}^{+} V(t, y)\right|_{y=x(t)}\right] .
\end{aligned}
$$

This proves (11).

The following lemma will be used in the proof of Theorem 7.

Lemma 6 (see Theorem 1.5.2 in [3]). Let $g \in C\left(\mathscr{R}_{+} \times \mathscr{R}_{+}, \mathscr{R}\right)$ and $r(t)$ be the maximal solution of

$$
\begin{aligned}
\dot{u} & =g(t, u), \\
u\left(t_{0}\right) & =u_{0},
\end{aligned}
$$

existing on $\left[t_{0}, \infty\right)$. Suppose $m \in C\left(\mathscr{R}_{+}, \mathscr{R}_{+}\right)$and $\operatorname{Dm}(t) \leq$ $g(t, m(t)), t \geq t_{0}$, where $D$ is any fixed Dini derivative. Then $m\left(t_{0}\right) \leq u_{0}$ implies $m(t) \leq r(t), t \geq t_{0}$.

Now we formulate the basic comparison results in terms of Lyapunov function $V$.

Theorem 7. Assume $\mathscr{D}^{+} V(t, x)$ satisfies

$$
\mathscr{D}^{+} V(t, x) \leq g(t, V(t, x)), \quad(t, x) \in \mathscr{R}_{+} \times \mathscr{R}^{n},
$$

where $g \in C\left(\mathscr{R}_{+}^{2}, R\right)$ is concave for $u$. Let $r(t)=r\left(t, t_{0}, u_{0}\right)$ be the maximal solution of the differential equation

$$
\begin{aligned}
\dot{u} & =g(t, u), \\
u\left(t_{0}\right) & =u_{0} \geq 0 .
\end{aligned}
$$

Then, for every solution of (1) $x(t)=x\left(t, t_{0}, x_{0}\right), V\left(t_{0}, x_{0}\right) \leq u_{0}$ implies

$$
\mathbb{E} V(t, x(t)) \leq r(t), \quad t \geq t_{0} .
$$

Proof. Denote $m(t)=\mathbb{E} V(t, x(t))$. By Lemma 5 and the concave of $g$ we have

$$
D^{+} m(t) \leq g(t, m(t)), \quad m\left(t_{0}\right) \leq u_{0} .
$$

By Lemma 6, we can obtain the result (27).

Remark 8. If $V \in C^{1,2}\left(\mathscr{R}_{+} \times \mathscr{R}^{n}, \mathscr{R}_{+}\right)$, the inequality (25) became

$$
\mathscr{L} V(t, x) \leq g(t, V(t, x)), \quad(t, x) \in \mathscr{R}_{+} \times \mathscr{R}^{n} .
$$




\section{Stability Criteria in terms of Two Measures}

Now we discuss the two-measure stability criteria for the stochastic differential system (1). We assume $f(t, 0)=$ $\sigma(t, 0)=0$ for all $t \geq t_{0}$. Firstly, we give some definitions for stochastic stability.

Definition 9. The stochastic differential system (1) is said to be

$\left(S_{1}\right)$ mean $\left(h_{0}, h\right)$-equistable, if for each $\epsilon>0$ and $t_{0} \epsilon$ $\mathscr{R}_{+}$, there exists a function $\delta=\delta\left(t_{0}, \epsilon\right)>0$ which is continuous in $t_{0}$ for each $\epsilon$ such that

$$
\mathbb{E} h_{0}\left(t_{0}, x_{0}\right)<\delta \quad \text { implies } \mathbb{E} h(t, x(t))<\epsilon, t \geq t_{0},
$$

where $x(t)=x\left(t, t_{0}, x_{0}\right)$ is any solution of (1);

$\left(S_{2}\right)$ mean $\left(h_{0}, h\right)$-uniformly stable, if $\left(S_{1}\right)$ holds with $\delta$ being independent of $t_{0}$;

$\left(S_{3}\right)$ mean $\left(h_{0}, h\right)$-quasiequiasymptotically stable, if for each $\epsilon>0$ and $t_{0} \in \mathscr{R}_{+}$, there exist positive number $\delta_{0}=\delta_{0}\left(t_{0}\right)$ and $T=T\left(t_{0}, \epsilon\right)$ such that

$\mathbb{E} h_{0}\left(t_{0}, x_{0}\right)<\delta_{0}$

$$
\text { implies } \mathbb{E} h(t, x(t))<\epsilon, t \geq t_{0}+T \text {; }
$$

$\left(S_{4}\right)$ mean $\left(h_{0}, h\right)$-quasiuniform asymptotically stable if $\left(S_{3}\right)$ holds with $\delta_{0}$ and $T$ being independent of $t_{0}$;

$\left(S_{5}\right)$ mean $\left(h_{0}, h\right)$-asymptotically stable if $\left(S_{1}\right)$ holds and, given $t_{0} \in \mathscr{R}_{+}$, there exists a $\delta_{0}=\delta_{0}\left(t_{0}\right)>0$ such that

$$
\mathbb{E} h_{0}\left(t_{0}, x_{0}\right)<\delta_{0} \text { implies } \lim _{t \rightarrow \infty} \mathbb{E} h(t, x(t))=0 ;
$$

$\left(S_{6}\right)$ mean $\left(h_{0}, h\right)$-uniformly equiasymptotically stable, if $\left(S_{1}\right)$ and $\left(S_{3}\right)$ hold together;

$\left(S_{7}\right)$ mean $\left(h_{0}, h\right)$-uniformly asymptotically stable if $\left(S_{2}\right)$ and $\left(S_{4}\right)$ hold simultaneously;

$\left(S_{8}\right)$ mean $\left(h_{0}, h\right)$-unstable if $\left(S_{1}\right)$ fails to hold. paper:

The following classes of functions will be used in this $\mathscr{K}=\left\{a \in C\left(\mathscr{R}_{+}, \mathscr{R}_{+}\right): a(u)\right.$ is strictly increasing in $u$ and $a(0)=0\}$,

$\mathscr{C} \mathscr{K}=\left\{a \in C\left(\mathscr{R}_{+}^{2}, \mathscr{R}_{+}\right): a(t, s) \in \mathscr{K}\right.$ for each $\left.t\right\}$,

$\Gamma=\left\{h \in C\left(\mathscr{R}_{+} \times \mathscr{R}^{n}, \mathscr{R}_{+}\right): \inf h(t, x)=0\right\}$.

Definition 10. Let $h_{0}, h \in \Gamma$. Then, we say that $h_{0}$ is finer than $h$ if there exists a function $\phi \in \mathscr{C} \mathscr{K}$ such that $h(t, x) \leq$ $\phi\left(t, h_{0}(t, x)\right)$. Furthermore, if $\phi$ is independent of $t$ then we call $h_{0}$ uniformly finer than $h$.

Definition 11. Let $V \in C\left(\mathscr{R}_{+} \times \mathscr{R}^{n}, \mathscr{R}_{+}\right)$. If there exists a function convex $b \in \mathscr{K}$ such that $b(h(t, x)) \leq V(t, x)$, then we call $V h$-positive definite. If there exists a concave function $a \in \mathscr{K}$ such that $V(t, x) \leq a(h(t, x))$, then we call $V h$ decrescent.
Theorem 12. Assume that

$\left(A_{0}\right) h_{0}, h \in \Gamma$ and $h_{0}$ is uniformly finer than $h$,

$\left(A_{1}\right) V \in C\left(\mathscr{R}_{+} \times \mathscr{R}^{n}, \mathscr{R}_{+}\right), V(t, x)$ satisfies (6), and $V$ is $h$-positive definite and $h_{0}$-decrescent,

$\left(A_{2}\right) g \in C\left(\mathscr{R}_{+}^{2}, \mathscr{R}\right)$ and $g(t, 0) \equiv 0$,

$\left(A_{3}\right) \mathscr{D}^{+} V(t, x) \leq g(t, V(t, x))$ for $(t, x) \in \mathscr{R}_{+} \times \mathscr{R}^{n}$.

Then, the stability properties of the trivial solution of (26) imply the corresponding $\left(h_{0}, h\right)$-stability properties of (1).

Proof. Since $V$ is $h$-positive definite, so there exists a convex $b \in \mathscr{K}$ such that

$$
b(h(t, x)) \leq V(t, x), \quad(t, x) \in \mathscr{R}_{+} \times \mathscr{R}^{n} .
$$

Suppose the trivial solution of (26) is equistable and $r\left(t, t_{0}, r\left(t_{0}\right)\right)\left(t \geq t_{0}\right)$ is its maximal solutions with initial time $t_{0}$ and initial value $r\left(t_{0}\right) \geq 0$, then, for every $\epsilon>0$, there exists $\delta_{1}>0$, when $r\left(t_{0}\right)<\delta_{1}$,

$$
r(t)=r\left(t, t_{0}, r\left(t_{0}\right)\right)<b(\epsilon), \quad t \geq t_{0} .
$$

Let $r\left(t_{0}\right)=\mathbb{E} V\left(t_{0}, x\left(t_{0}\right)\right)$; then, by Theorem 7 , we have

$$
\mathbb{E} V(t, x(t)) \leq r(t), \quad t \geq t_{0} .
$$

Since $V$ is $h_{0}$-decrescent, there exists a concave $a \in \mathscr{K}$ such that

$$
V(t, x) \leq a\left(h_{0}(t, x)\right), \quad(t, x) \in \mathscr{R}_{+} \times \mathscr{R}^{n} .
$$

So

$$
r\left(t_{0}\right) \leq \mathbb{E} a\left(h_{0}\left(t_{0}, x\left(t_{0}\right)\right)\right) \leq a\left(\mathbb{E} h_{0}\left(t_{0}, x\left(t_{0}\right)\right)\right) .
$$

Since $a$ is continuous and strictly increasing, so let $\delta=$ $a^{-1}\left(\delta_{1}\right)$; then when $\mathbb{E} h_{0}\left(t_{0}, x\left(t_{0}\right)\right)<\delta$, inequality (34) holds. Combining (33), (34), and (35) and using the strictly increase of $b$, we can gain

$$
\mathbb{E} h\left(t, t_{0}, x\left(t_{0}\right)\right)<\epsilon, \quad t \geq t_{0},
$$

which implies $(1)\left(h_{0}, h\right)$-equistability.

Remark 13. If $V \in C^{1,2}\left(\mathscr{R}_{+} \times \mathscr{R}^{n}, \mathscr{R}_{+}\right)$, then condition $\left(A_{3}\right)$ can be replaced by

$$
\mathscr{L} V(t, x) \leq g(t, V(t, x)) .
$$

Remark 14. The stabilities of auxiliary ordinary differential equation (26) are defined by Definition 2.4.1 in [3].

Example 15. Consider the following 2-dimensional Itô's system:

$$
\begin{aligned}
d x_{1}(t)= & x_{2}(t) d t \\
d x_{2}(t)= & -\left(b x_{1}(t)+a x_{2}(t)\right) d t \\
& +\left(c x_{2}(t)+e x_{1}(t)\right) d B_{t} .
\end{aligned}
$$


Let $h(t, x)=h_{0}(t, x)=e^{\gamma t}\left(x_{1}^{2}+x_{2}^{2}\right)$; suppose $V(t, x)$ has the form

$$
V(t, x)=e^{\gamma t}\left(\alpha x_{1}^{2}+2 \beta x_{1} x_{2}+x_{2}^{2}\right)
$$

Then

$$
\begin{aligned}
\mathscr{L} V & =e^{\gamma s}\left[\left(\gamma \alpha-2 \beta b+e^{2}\right) x_{1}^{2}\right. \\
& +(2 \gamma \beta+2 \alpha-2 \beta a-2 b+2 c e) x_{1}(s) x_{2} \\
& \left.+\left(\gamma+2 \beta-2 a+c^{2}\right) x_{2}^{2}\right] .
\end{aligned}
$$

In order to make $V(t, x) h$-positive, we let

$$
\begin{array}{r}
\alpha>0, \\
\alpha-\beta^{2}>0
\end{array}
$$

Let $g(t, u)=-\theta u, \theta>0$; in order to find $\theta>0$ to satisfy

$$
\mathscr{L} V \leq g(t, V)
$$

we set

$$
\begin{array}{r}
2 \beta \gamma+2 \alpha-2 \beta a-2 b+2 c e=0, \\
\gamma \alpha-2 \beta b+e^{2}<0, \\
\gamma+2 \beta-2 a+c^{2}<0 .
\end{array}
$$

When $\gamma<a$, combining (43) and (45), we have that, when

$$
\begin{aligned}
& \max \left\{\frac{1}{2}\left[a-\gamma-\sqrt{(\gamma-a)^{2}+4(b-c e)}\right],\right. \\
& \left.\frac{\gamma b-\gamma c e+e^{2}}{\gamma^{2}+2 b-\gamma a}\right\}<\beta<\min \left\{\frac{b-c e}{\gamma-a}, a\right. \\
& -\frac{1}{2}\left(\gamma+c^{2}\right), \\
& \left.\frac{1}{2}\left[a-\gamma+\sqrt{(\gamma-a)^{2}+4(b-c e)}\right]\right\},
\end{aligned}
$$

there exists $\theta>0$ which satisfies (44). Moreover, the trivial solution of

$$
\dot{u}=-\theta u
$$

is uniformly asymptotically stable; by Theorem 12 , the stochastic differential is mean- $\left(h_{0}, h\right)$-asymptotically stable.

However, in practice, the coefficients maybe have some uncertainty properties; that is, we only know the range of the parameters $a, b$. Then how to discuss the stability of such systems with uncertainty is still a very interesting problem. The following section will introduce an optimal control approach to discuss the stability of such systems with uncertainty.

\section{The Stability for Systems with Uncertainty}

Consider the following stochastic differential equations with uncertainty:

$$
d x=[f(x)+g(x) k(x)] d t+\sigma(x) d B_{t},
$$

where $k(x)$ is an uncertainty function, and $f(0)=k(0)=$ $\sigma(0)=0$, that is, the trivial solution, is 0 of (48). Furthermore, we also suppose there exists a function $k_{\max }(x) \geq 0$ such that

$$
\|k(x)\| \leq k_{\max }(x) .
$$

Now we discuss how to determine the asymptotic stability of system (48) for all uncertainty function $k$. Similar to the methods applied by $[18,19]$, we can translate this stability problem into an optimal problem.

For the nominal system

$$
d x=[f(x)+g(x) u] d t+\sigma(x) d B_{t}
$$

suppose we can find a state-feedback control $u=\bar{u}(x)$ that minimizes the cost functional

$$
J(x(\cdot), u)=\mathbb{E}^{y} \int_{0}^{\infty}\left[f_{\max }^{2}(x)+\rho h_{0}(x)+3 u^{T} u\right] d t,
$$

where $h_{0} \in C\left(\mathscr{R}^{n}, \mathscr{R}_{+}\right), y$ is the initial value of (50), $\rho>0$, and $u$ is admissible on $[0, T]$ for each $T<\infty$, and satisfies

$$
J(x(\cdot), u)<\infty .
$$

Let the value function

$$
V(y)=\min _{u} J(x(\cdot), u) .
$$

Theorem 16. Suppose $\bar{u}(x)$ is an optimal control of problem (53), and there exists $\bar{\rho} \leq \rho$ satisfying

$$
\|\bar{u}\|^{2} \leq \frac{\bar{\rho}}{2} h_{0}(x),
$$

and the value function $V \in C^{2}\left(\mathscr{R}^{n}, \mathscr{R}_{+}\right), V$ is also $h$ positive, and $h_{0}$ is decrescent, then system (48) is uniformly mean $\left(h_{0}, h\right)$ uniformly asymptotically stable for all uncertainties $k(x)$.

Proof. The values function $V$ satisfies the Hamilton-JacobiBellman equation:

$$
\begin{gathered}
\min _{u \in \mathscr{R}^{p}}\left(f_{\max }^{2}+x^{T} x+u^{T} u+\left\langle V_{x}, f+g u\right\rangle\right. \\
\left.+\frac{1}{2}\left\langle\sigma \sigma^{T}, V_{x x}\right\rangle\right)=0 .
\end{gathered}
$$

So, the optimal control $\bar{u}$ satisfies

$$
\begin{aligned}
& f_{\max }^{2}+x^{T} x+\bar{u}^{T} \bar{u}+\left\langle V_{x}, f+g \bar{u}\right\rangle+\frac{1}{2}\left\langle\sigma \sigma^{T}, V_{x x}\right\rangle \\
& \quad=0 \\
& 2 \bar{u}^{T}+V_{x}^{T} g=0 .
\end{aligned}
$$


Then, the Lyapunov generator of (48) for $V$ is given as

$$
\begin{aligned}
\mathscr{L} V(x) & :=\left\langle V_{x}, f+g k\right\rangle+\frac{1}{2}\left\langle V_{x x}, \sigma \sigma^{T}\right\rangle \\
& =\left\langle V_{x}, g k\right\rangle-\left\langle V_{x}, g \bar{u}\right\rangle-h_{0}(x)-k_{\max }^{2}-\bar{u}^{T} \bar{u} \\
& \leq\|k\|^{2}-k_{\max }^{2}-(\rho-\bar{\rho}) h_{0}(x) \\
& \leq-(\rho-\bar{\rho}) h_{0}(x) .
\end{aligned}
$$

So, for the solutions $x(t)$ of (48) with uncertainty $k(x)$, applying Itô's formula to $V(x(t))$, we have, when $t \geq t_{0}$,

$$
\begin{aligned}
& \mathbb{E} V(x(t))-\mathbb{E} V\left(x\left(t_{0}\right)\right)=\mathbb{E} \int_{t_{0}}^{t} \mathscr{L} V(x(s)) d s \\
& \quad \leq-(\rho-\bar{\rho}) \mathbb{E} \int_{t_{0}}^{t} h_{0}(x) d s .
\end{aligned}
$$

Hence

$$
\mathbb{E} V(x(t)) \leq \mathbb{E} V\left(x\left(t_{0}\right)\right)-\mathbb{E} \int_{t_{0}}^{t} h_{0}(x(s)) d s,
$$

$\forall t \geq t_{0}$.

So $m(t)=\mathbb{E} V(x(t))$ is decreasing on $[0, \infty]$. Now we show $m(t) \rightarrow 0$ when $t \rightarrow \infty$. Suppose $m(t) \rightarrow m_{0}>0$. Since $V$ is $h_{0}$ decrescent, so there exists $c>0$ and $t_{0}>0$, such that $\mathbb{E} h_{0}(x(t))>c>0\left(t \geq t_{0}\right)$ combining with (59); we have

$$
\begin{aligned}
\mathbb{E} V(x(t)) \leq \mathbb{E} V\left(x\left(t_{0}\right)\right)-c\left(t-t_{0}\right) & \longrightarrow-\infty, \\
t & \longrightarrow \infty .
\end{aligned}
$$

This contradicts the fact that $V(x) \geq 0$. This implies that system $(48)$ is uniformly mean $\left(h_{0}, h\right)$ uniformly asymptotically stable.

Corollary 17. Specially, let

$$
\begin{aligned}
f(x) & =A x, \\
g(x) & =G, \\
\sigma(x) & =C x, \\
k_{\max }(x) & =M\|x\| .
\end{aligned}
$$

The value function can be given by $V(x)=x^{T} P x$ and the optimal control $\bar{u}(x)=-G^{T} P x$, where $P>0$ satisfies the following Riccati equation:

$$
\left(M^{2}+1\right) I+P A+A^{T} P+C^{T} P C-P G G^{T} P=0 .
$$

Let $\lambda_{\max }$ be the maximal eigenvalue of $P G G^{T} P$. If $\lambda_{\max }^{2} \leq \rho / 2$, then the corresponding system with uncertainty is uniformly mean $\left(h_{0}, h\right)$-equistable for all uncertainties $k(x)$ (which is also mean square asymptotically stable for all uncertainties $k(x)$ ).

Example 18. Consider (41) with uncertainty coefficients. In (41), replace $a, b$ by $a+\sin x_{1}$ and $b+\cos x_{2}$, respectively, and $a$ takes values in $[1.4,1.6], b$ in $[0.9,1.1], c=-1$, and $e=1$. Then the system with uncertainty is obtained:

$$
\begin{aligned}
& d x_{1}(t)=x_{2}(t) d t \\
& d x_{2}(t)=-\left[\left(b+0.1 \cos B_{t}\right) x_{1}(t)\right. \\
& \left.\quad+\left(a+0.1 \sin B_{t}\right) x_{2}(t)\right] d t+\left(c x_{2}(t)+e x_{1}(t)\right) d B_{t} .
\end{aligned}
$$

Let

$$
\begin{aligned}
& A=\left[\begin{array}{cc}
0 & 1 \\
-1 & -1.5
\end{array}\right], \\
& B=\left[\begin{array}{cc}
0 & 0 \\
1.5-b-0.1 \cos B_{t} & 1-a-0.1 \sin B_{t}
\end{array}\right], \\
& C=\left[\begin{array}{cc}
0 & 0 \\
1 & -1
\end{array}\right],
\end{aligned}
$$

And let $G$ be the 2-order identical matrix. Let $h(x)=h_{0}(x)=$ $\|x\|^{2}$; then

$$
\begin{aligned}
& f(x)=A x, \\
& g(x)=G, \\
& k(x)=B x, \\
& \sigma(x)=C x .
\end{aligned}
$$

We can take $k_{\max }(x)=0.3 \sqrt{x_{1}^{2}+x_{2}^{2}}$ and the auxiliary optimal problem is

$$
d x=(A x+G u) d t+C x d B_{t},
$$

with the cost functional

$$
J(x(\cdot), u)=\mathbb{E}^{y} \int_{0}^{\infty}\left[3 x^{T} x+u^{T} u\right] d t,
$$

$\rho=2.91$, solving (62) with $M=0.3$; we have

$$
P=\left[\begin{array}{cc}
1 & 0 \\
0 & 0.5
\end{array}\right] .
$$

By Corollary 17, we can determine that the stochastic system (63) is uniformly mean $\left(h_{0}, h\right)$ uniformly stable for all uncertainties.

\section{Conclusion}

In this paper, we extend the stability criteria of two measures to the mean stability situations for the stochastic systems with uncertainty. For the usual SDE, we give the results of mean stability criteria which are the basic criteria for such systems. As far as the systems with uncertainty, in order to resolve the difficulties coming from the coefficient uncertainty, we use the optimal control results as an auxiliary method to determine the mean stability. Furthermore, the stability criteria in terms of two measures for other stochastic systems, such as systems with Markovian jumps or Poisson jumps, are worth further studying. 


\section{Conflict of Interests}

The authors declare that there is no conflict of interests regarding the publication of this paper.

\section{Acknowledgments}

This work was supported by the NSF of China (61471224, 61402265, and 11271007), the NSF of Shandong Province (ZR2011FM018), and the Doctoral Fund of Ministry of Education (20123718110010).

\section{References}

[1] A. M. Lyapunov, The General Problem of the Stability of Motion, translated by A. T. Fuller, Taylor \& Francis, 1992 (Russian).

[2] A. A. Movchan, "Stability of processes with respect to two measures," Prikladnaia Matematika i Mekhanika, vol. 24, pp. 988-1001, 1960.

[3] V. Lakshmikantham, S. Leela, and A. A. Martynyuk, Stability Analysis of Nonlinear Systems, Marcel Dekker, 1989.

[4] H. J. Kushner, Stochastic Stability and Control, Academic Press, 1967.

[5] R. Z. Has'minskii, Stochastic Stability of Differential Equations, translated by D. Louvish, Sijthoff and Noordhoff, 1981.

[6] L. Arnold, Stochastic Differential Equations: Theory and Applications, John Wiley \& Sons, 1974.

[7] Q. Zhu, "Asymptotic stability in the pth moment for stochastic differential equations with Lévy noise," Journal of Mathematical Analysis and Applications, vol. 416, no. 1, pp. 126-142, 2014.

[8] X. Mao, Exponential Stability of Stochastic Differential Equations, Marcel Dekker, 1994.

[9] X. Mao, "Stochastic versions of the LaSalle theorem," Journal of Differential Equations, vol. 153, no. 1, pp. 175-195, 1999.

[10] Q. Zhu, "Stabilization of stochastically singular nonlinear jump systems with unknown parameters and continuously distributed delays," International Journal of Control, Automation and Systems, vol. 11, no. 4, pp. 683-691, 2013.

[11] N. Rouche, P. Habets, and M. Laloy, Stability Theory by Liapunov's Direct Method, Springer, 1977.

[12] H. Wang and Q. Zhu, "Finite-time stabilization of high-order stochastic nonlinear systems in strict-feedback form," Automatica, vol. 54, pp. 284-291, 2015.

[13] Y. Xu and X. Chen, "Rules mining of power system transient stability assessment based on clustering analysis and rough set theory," Applied Mechanics and Materials, vol. 521, pp. 418-422, 2014.

[14] Q. Zhu, J. Cao, and R. Rakkiyappan, "Exponential input-to-state stability of stochastic Cohen-Grossberg neural networks with mixed delays," Nonlinear Dynamics, vol. 79, no. 2, pp. 1085-1098, 2015.

[15] C. Yuan, "Stability in terms of two measures for stochastic differential equations," Dynamics of Continuous, Discrete and Impulsive Systems Series A: Mathematical Analysis, vol. 10, no. 6, pp. 895-910, 2003.

[16] W. Grote, D. Kastsian, and M. Mönnigmann, "Guaranteed stability in optimal power generation dispatching under uncertainty," IEEE Transactions on Power Systems, vol. 28, no. 2, pp. 1103-1112, 2013.
[17] X. Mao, Stochastic Differential Equations and Applications, Horwood Publishing, 2007.

[18] F. Lin, R. D. Brandt, and J. Sun, "Robust control of nonlinear systems: compensating for uncertainty," International Journal of Control, vol. 56, no. 6, pp. 1453-1459, 1992.

[19] F. Lin, "An optimal control approach to robust control design," International Journal of Control, vol. 73, no. 3, pp. 177-186, 2000. 


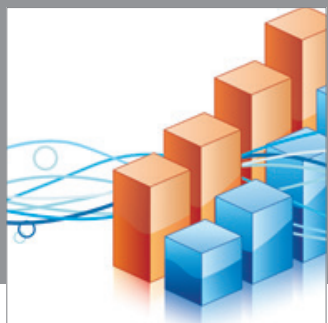

Advances in

Operations Research

mansans

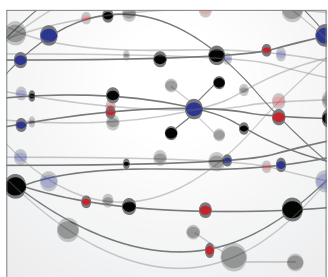

The Scientific World Journal
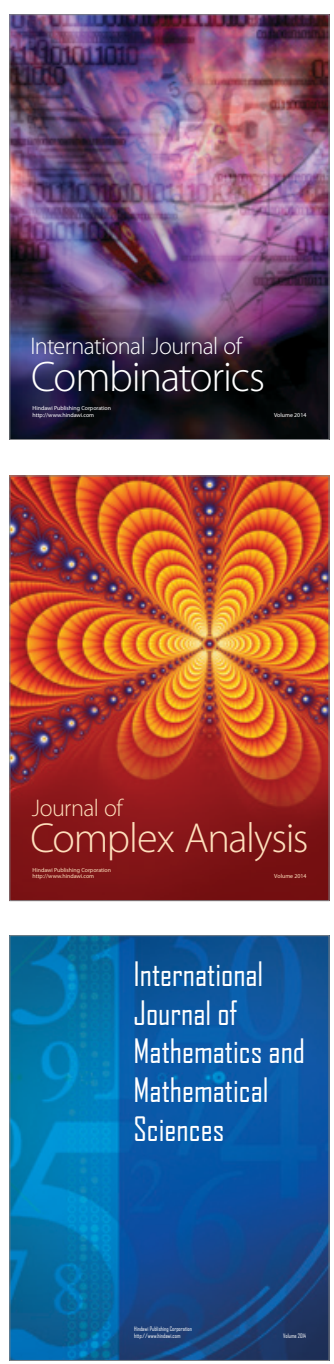
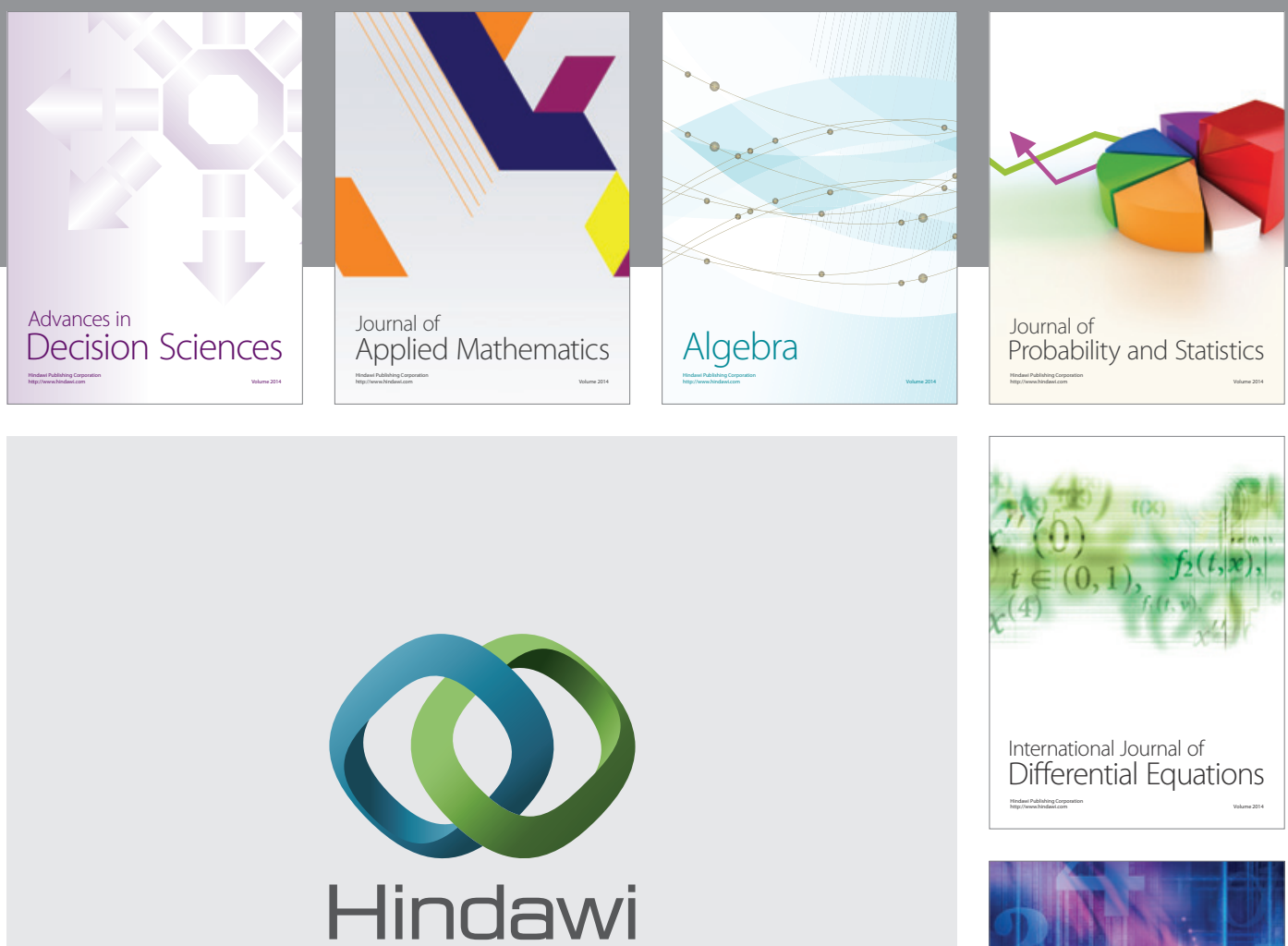

Submit your manuscripts at http://www.hindawi.com
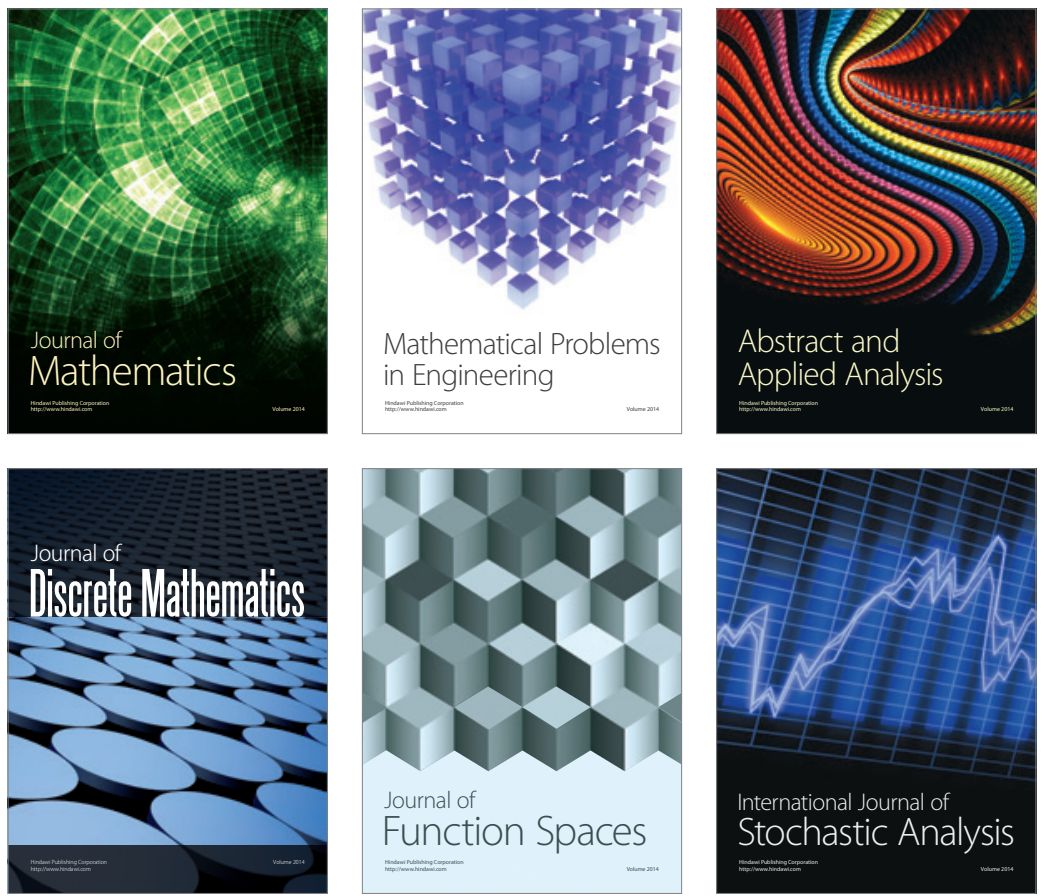

Journal of

Function Spaces

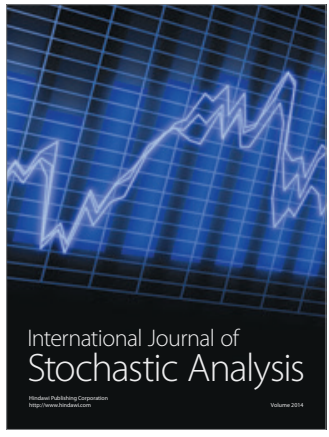

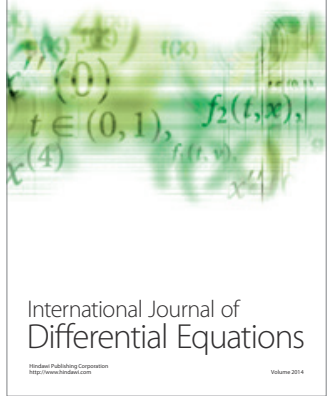
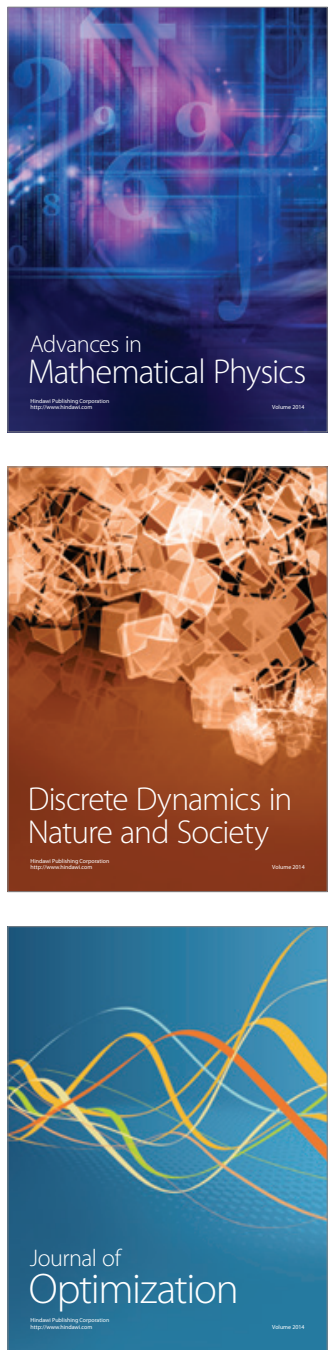